The papers appearing below are based upon verbal communication delivered at a meeting of the Scottish Area B.A.S.M. and are not therefore written in the style usual for printed scientific articles.

$E d$.

\title{
RESEARCH METHODOLOGY IN BIOENGINEERING AND APPLIED HUMAN PHYSIOLOGY
}

\author{
JAMES MACGREGOR, M.Sc., Ph.D.
}

A definition of BioEngineering is that it is the application of the latest techniques of science and technology to the practical solution of significant clinical problems.

Sports Medicine, interested as it is in the assessment of human performance, stands to gain from advances in BioEngineering as a result of the development of practical investigative techniques and the availability of powerful research methods. Both clinical problems and sports problems are often resolved by the application of adequate techniques of assessing the performance of the human subject in biochemical, biomechanical or even mathematical terms.

Assessment of human performance requires a planned approach undertaken in a series of sequential yet distinct stages, namely:

1. Identification of the problem area and definition of the probable interaction of those parameters to be investigated.

2. Organisation of a survey, trial, investigation etc. to obtain both retrospective and prospective data in such a way that in the long run any inferences drawn from results can be considered to be adequately controlled from a statistical point of view.

3. Design and development of new transducers or systems for obtaining quantitative information about particular aspects of the subjects performance and transposing it to an appropriate form of record for subsequent analysis.

4. Organisation of the purely administrative aspects of the investigation, which are often overlooked, bearing in mind both safety requirements and the social needs of the patient. The legitimate professional interest of medical advisers, coaches etc. must be catered for at this point.
5. Adequate processing of the information obtained from the enquiry with the use of effective analytical methods which results in the calculation of suitable statistical measures of the relevant variables.

6. The effective interpretation of the results of the analysis with particular care taken to incorporate in to a prepared statement any restrictionso imposed by the limitations of the methods used during the investigation as a result of clinicalo requirements, practical expediency etc.

A surprisingly large part of effective research is related to effective planning and administration. Only when this area is adequately covered can one expect to reap the full benefit of the professional expertise now available in the fields of mathematics, statistics, electronics, biomechanics, biophysics etc.

Particular care has to be taken to ensure that the apparent significance of purported results are not overexaggerated when presented to individuals with no specific technical competence in the area. The commonest fault in research results published today is the overemphasis on possible significance and under emphasis on the limitations. This is perhaps a natural development in a world dedicated to sales promotion, market research and the award of accolades to the most plausible.

The series of papers to be presented explores the application of mechanics, statistics, and electronics to problems of human performance and highlights the difficulties which must be overcome for adequate investigation. They also serve to explain why the adequate prosecution of research in sport physiology usually requires very extensive facilities and budgets. 